\title{
APLICACIONES DE LA MENTALIZACIÓN CON FAMILIAS EN CONTEXTOS DE PROTECCIÓN Y JUSTICIA JUVENIL ${ }^{1}$
}

\author{
Ángel Estalayo Hernández ${ }^{2}$, Olga Rodríguez Ochoa ${ }^{3}$ \\ y Raúl Gutiérrez Sebastián ${ }^{4}$ \\ Zaragoza
}

\begin{abstract}
El desarrollo de la terapia basada en la mentalización, cada día más considerada como una concepción transversal en todas las modalidades de psicoterapia, dispone de varias aplicaciones, más allá del abordaje individual. En este texto se plantea una propuesta de intervención con similitudes técnicas con la mentalización focalizada en el trabajo con familias. De igual forma se presentará esta modalidad dentro de contextos que conllevan involuntariedad, entendiendo que posibilita especialmente la intervención. Todo ello, desde una concepción circular de las relaciones y puntuada desde la interpretación de los estados mentales. Para ello, se evalúan las distintas formas inadecuadas de la capacidad individual de mentalizar (modos pre-mentalizadores) y su impacto en la dinámica relacional y también cómo la dinámica relacional construida impacta, filtra y rigidifica la capacidad mentalizadora de cada miembro del sistema en relación, generando un ambiente invalidante. A continuación, se mencionarán las técnicas clasificadas según las dificultades en una propuesta de diagnóstico relacional
\end{abstract}

Palabras clave:Justicia juvenil, Protección, Mentalización, Familia, Violencia, Psicoterapia de Apoyo.

The development of mentalization based therapy increasingly transcends multiple forms of psychotherapy. This consists of various methods, which go beyond the individual approach. This paper will propose intervention of several similar techniques whereby the mentalization will be focused on working with families. Likewise these methods will be presented within contexts involving involuntary behaviour, on the assumption that said methods will facilitate intervention. All this is based upon a circular conception of relationships and scored on the basis of the interpretation of mental states. To achieve this, evaluations must be made, of the various inappropriate methods of the individual's capacity to mentalize, (pre-meditators). Its impact on the relative dynamic must also be considered, that, once built up, how it proceeds to inhibit the mentalizing capacity of every member of the system related to it, thereby crippling the surrounding environment.Following on from this, we will examine the listed techniques related to the problems in a proposal for relational diagnostics.

Key Words: Juvenile justice, Protection of minors, Mentalization, Family, violence, supportive psychotherapy.

English Title:Applying Mentalization with Families in Juvenile Justice and Protection Contexts.

\section{Cita bibliográfica / Reference citation:}

Estalayo, Ángel; Rodriguez, Olga y Gutiérrez, Raúl. (2017). Aplicaciones de la Mentalización con Familias en Contextos de Protección y Justicia Juvenil. Clínica e Investigación Relacional, 11 (1): 110133. [ISSN 1988-2939] [Recuperado de www.ceir.info ] DOI: 10.21110/19882939.2017.110107

\footnotetext{
${ }^{1}$ Trabajo basado en el texto presentado en las IV jornadas de Psicoanálisis Relacional, organizadas por el Instituto de Psicoterapia Relacional(Salamanca, 28-29 octubre de 2016)

${ }^{2}$ Director técnico IRSE-EBI. Psicoterapeuta FEAP.Email:aestaher@irse-ebi.org

3 Psicólogo y Psicoterapeuta FEAP.Email: olgaotosoa@yahoo.es

4 Psicólogo en FAIM. Psicoterapeuta FEAP.Email: raulgs@cop.es
}

\section{CeIR Vol. 11 (1) - Febrero 2017 ISSN 1988-2939 - www.ceir.info}

(c) Derechos reservados/Copyright de Clínica e investigación Relacional y los autores. Prohibida la reproducción total o parcial sin autorización expresa. Este material es para uso científico y profesional exclusivamente y puede contener información clínica sensible. Los editores no se responsabilizan de los contenidos de los autores. Dirigir las consultas sobre derechos y autorizaciones a ceir@psicoterapiarelacional.es 


\section{1.-YA SE HACE MENTALIZACIÓN CON FAMILIAS.}

La idea de trabajar con familias desde la mentalización ya existe. Eia Asen propone un nuevo enfoque de la terapia familiar basado en la mentalización (MBT-F, Asen y Fonagy; Fearcon et al 2006), encontrando similitudes técnicas con corrientes estructurales. Existe cierto esfuerzo actual de aplicar este concepto de la mentalización al mundo sistémico en general, proponiendo así un enfoque que trata de integrar el mundo interno psicodinámico y el mundo externo sistémico. Assen plantea la posibilidad de mejorar la eficacia de la terapia sistémica ayudando a la familia a dar sentido a los sentimientos que son experimentados por cada miembro de la familia, el pensamiento que está conectado con estos sentimientos, cómo estos sentimientos se comunican dentro de la familia y cómo la falta de comunicación o los malentendidos de estos sentimientos puede alimentar las interacciones que mantienen los problemas familiares. Este nuevo enfoque de la terapia familiar basado en la mentalización (MBT-F, Asen y Fonagy; Fearcon et al 2006) ha comenzado a vincular los mundos intra-personales e interpersonales. Este enfoque asume que muchos problemas en las familias se reducirán si las habilidades de los miembros de la familia para pensar acerca de los estados mentales de otros se promueven y liberan de obstáculos. La mentalización enfocada en terapia sistémica (MBT-F) se centra en los pensamientos y sentimientos de cada miembro de la familia y en las relaciones entre ellos.

También cabe señalar a Bleiberg (2013) quién desarrolla una modalidad también basada en la mentalización con familias que tienen miembros adolescentes desde una perspectiva diferente y complementaria a la anterior.

Por otra parte, teniendo en cuenta que nuestra labor suele centrarse en procesos que presentan dificultades de vinculación, agresividad y/o violencia, así como conflicto con el mundo adulto y la autoridad, recogemos el concepto de "Confianza Epistémica" de Fonagy (2013) en el que confluyen el instinto del apego que conceptualizaron Bowlby y Ainsworth, así como el instinto de la comunicación de Gergely, Tronick y las ramas modernas de investigación infantil, basadas en la Pedagogía Natural. Todo ello nos permite atender el espacio seguro del apego y la confianza desde una perspectiva válida para la figura del profesional, pero sobre todo para el subsistema parental del sistema familiar de referencia de cada persona con la que nos relacionamos en nuestros contextos de intervención. 
En la misma línea entendemos el concepto de sintonía emocional validante de Stolorow, Orange y Atwood (2012), siendo de especial relevancia la forma de vincular entre el profesional y paciente, así como la forma de co-construir un acceso o reinterpretación de los distintos niveles de inconsciente que describen, tanto en el caso de aquellas emociones que fueron reprimidas por la respuesta del entorno, como aquellas que ni tan siquiera evocaron respuesta o reacción alguna. Tanto es así, que da nombre a nuestra metodología concreta de intervención vinculación Emocional Validante (VEV) de Estalayo, Rodríguez y Romero (2009).

\section{2.-NUESTRA PROPUESTA (VEV).}

No es el propósito exponer la metodología VEV de carácter integrador. Más bien, algunos aspectos que proceden y confluyen especialmente de la mentalización y de la perspectiva intersubjetiva, encajando a su vez perfectamente con algunas perspectivas sistémicas. De esta forma, creemos que se puede generar una sinergia de la mentalización con una perspectiva circular. Para establecer la interrelación existente entre la mentalización, la circularidad y el sistema, proponemos detectar la circularidad entre nuestros estados mentales y los del otro, y ver-entender cómo estas dinámicas circulares están en la base de los problemas y de las soluciones. Ello, para posteriormente puntuar el ambiente desde la parte más capaz, que es aquella que tiene más desarrollada la autoconciencia reflexiva, es decir, la capacidad de mentalización. Es por ello, que al tratar de cohesionar la mentalización como facultad individual y la circularidad como dinámica relacional, pueda obtenerse un abordaje que establezca formas de intervención circulares pero basadas en dinámicas mentalizadoras, es decir, en narrativas que permitan la confrontación o connotación positiva, cuando sea el caso, "darse cuenta" de los propios estados mentales y de los del resto de los miembros del sistema que se encuentran tras las conductas que originan malestar, "comprender" la interconexión entre los estados emocionales de los diferentes miembros, al igual que entender cómo la mala interpretación de los mismos puede ocasionar problemas de comunicación en la base de la disfunción familiar.

El trabajo terapéutico consiste en generar un clima o ambiente dentro del sistema que genere la posibilidad entre los miembros de "verse" más allá de los síntomas o conductas, completar la interpretación de las mismas y abrir nuevas vías de comunicación. 
Desde este enfoque proponemos simultanear lo individual y dimensional con lo relacional y ello conlleva establecer el foco de la intervención sobre las dinámicas relacionales que se dan entre los miembros familiares y desde los estados emocionales de los pacientes y el terapeuta.

Ello se puede concretar en:

1) Evaluar el modo predominante de pensamiento en la familia (equivalencia psíquica, modo como si, modo teleológico), el nivel de comunicación y la capacidad de mentalización.

2) Medir o valorar las diferencias individuales de los miembros a través de los cuatro polos de la mentalización, lo cual nos ayudará para identificar al miembro más capaz.

3) Evaluar al miembro más capaz y al paciente designado del sistema para calcular la zona de desarrollo óptimo entre el paciente identificado y el miembro más capaz. Ayudar al tránsito evolutivo del paciente identificado.

4) Generar un espacio terapéutico seguro, en el cual las actitudes del terapeuta mentalizador son fundamentales.

5) Analizar la demanda que la familia trae a terapia. Reformular la misma en clave de relación. Devolver una carta de reformulación a toda la familia: en la que se ha recogido su objetivo y se ha reformulado en clave relacional y con una propuesta de visión mentalizadora de la relación. Hacer una descripción relacional del síntoma.

6) Hacer una propuesta de trabajo centrada en el proceso y no tanto en el contenido con el propósito de abrir canales comunicativos para que ellos encuentren la solución. Entender que lo que la familia es capaz de hacer en sesión con nosotros, son capaces de hacerlo fuera porque ya encontrarán ellos las soluciones. Una vez que la familia aprende a verse, las soluciones las tiene que encontrar la propia familia.

7) Utilizar como estrategia básica la interrupción o mano mentalizadora cada vez que se pierde la capacidad de mentalización y recuperarla desde la identificación de la emoción básica cuya aparición intensa ha ocasionado dicha pérdida de reflexividad.

8) Una vez que la mentalización está restaurada y la familia "se ve" se puede trabajar técnicas específicas de terapia familiar (roles, reglas...).

9) Partir de que el formato de trabajo no tiene por qué ser con todo el sistema a la vez, puede ser con subsistemas o individuos concretos, entendiendo que la clave es 
dónde y cómo se centra la atención.

10) Partir de la importancia de la persona del profesional y del desarrollo de consciencia de que puede hacer intervenciones basadas en su propia pérdida de mentalización. Nuestra inhabilidad de comunicarnos con nuestros pacientes nos causa frustración y la tendencia a culpar al paciente. Sentimos que no nos escuchan, pero en realidad sucede que para ellos es difícil confiar en lo que oyen (Fonagy, 2013).

\section{UNAS PALABRAS SOBRE LOS CONTEXTOS DE INVOLUNTARIEDAD: JUSTICIA JUVENIL Y PROTECCIÓN}

La característica principal del trabajo en contextos de protección y justicia juvenil es precisamente la intervención con pacientes involuntarios sin una demanda explicita ni conciencia de problema.

Esto nos exige generar dinámicas de relación desde donde tomar conciencia de determinadas dificultades y abordar las mismas con el menor, con el grupo de convivencia, como sistema significativo de referencia, y con la propia familia.

Otro rasgo a reseñar de esta tipología de población es precisamente la prevalencia de problemas de salud mental que confirman determinadas investigaciones. Rioseco (2009), habla de cómo se identifica una elevada presencia de patología psiquiátrica y se observa una mayor presencia de trastornos psicopatológicos en el grupo de jóvenes infractores, con una elevada prevalencia de trastorno disocial y abuso de sustancias y una menor capacidad intelectual. El $16 \%$ de los adolescentes institucionalizados fue evaluado con una inteligencia normal promedio y el $78 \%$ está dentro de categorías de clasificación que reflejan un potencial intelectual deficitario.

Además, la intervención no es llevada a cabo por un único agente, sino que el menor convive con un equipo interdisciplinar que atiende las dificultades del menor de manera intensiva a lo largo de su estancia en el centro, lo que insta a establecer cohesión en cuanto a la diversidad de criterios y formas de intervenir, atendiendo precisamente a los conflictos propios de cada menor y las características definitorias de cada profesional al servicio del proceso de cambio del adolescente.

De lo anteriormente expuesto, entendemos que intervenir con este tipo de población, requiere definir procesos de intervención que aborden, no solo las dificultades propias del adolescente sino del sistema relacional originario que pueda sostener procesos 
madurativos del menor.

Es por ello que entendemos las aportaciones de la mentalización como muy relevantes, partiendo de las características de nuestro contexto que pueden tener forma de una psicoterapia de apoyo entre lo clínico y educativo-asistencial, de una propuesta educativo-terapéutica que se basa en generar adhesión a la relación de ayuda, toma de conciencia de problema y de los recursos propios, capacidad autorregulatoria, de autorreflexión y responsabilización, remisión de sintomatología aguda, y promover el tránsito al espacio convivencial normalizado. Además, aspectos como la involuntariedad o el peligro de fijar el síntoma en una dinámica relacional que adhiera la misma a una identidad en proceso en una etapa evolutiva como adolescencia, también encuentran respuesta en la "confianza epistémica", o en las etapas que proponen (apoyo, clarificación, mentalización de las emociones y mentalización de la trasferencia) que se corresponden con nuestra propuesta Estalayo, Rodríguez, Romero (2009). De esta forma, las aportaciones de la mentalización y de sus conexiones conceptuales y metodológicas con la concepción sistémica e intersubjetiva, nos resultan especialmente útiles. Máxime, teniendo en cuenta:

1. El cambio en las características de la población con la que nos relacionamos; aumento de prevalencia de aspectos relacionados con problemas de salud mental y el papel del entorno en su etiología y posibilidad de contención.

2. Que pueden concretarse en capacidad autorregulatoria, capacidad reflexiva, retrasos madurativos y capacidad de responsabilización.

3. Que no participan voluntariamente en estos procesos de ayuda.

4. Que los cambios tienen múltiples causas que pueden concentrarse y subrayar la importancia de la relación educativo-terapéutica y de las características personales del profesional.

5. $Y$ que existen factores comunes estudiados como fuentes de cambio (las características personales del profesional de intervención directa, el trabajo con la familia, la coordinación interinstitucional y disponer de un modelo o comprensión conjunta que guie la intervención...).

Por otra parte, la violencia como factor externalizante forma parte de nuestra práctica: su expresión, comprensión y forma de intervención son uno de los ejes de estos contextos de intervención. Habida cuenta de ello, el papel de la mentalización en la violencia es otro argumento de la aplicabilidad de esta perspectiva en Justicia Juvenil y 
Protección. De esta forma, bien porque los individuos sin un sentido de la identidad bien definida pueden sentir fácilmente que no son responsables de sus propias acciones, bien por las dificultades que pueden presentar en la anticipación de las consecuencias, o bien por las variaciones del sistema representacional que conlleva que los sentimientos o pensamientos no se experimenten como reales o con sentido, se nos hace necesario incorporar la mentalización.

También la diferencia entre afecto instrumental o manipulativo en lugar del comunicativo (Fonagy y Target, 1999), nos resulta útil para diferenciar e intervenir en posteriores actos de violencia con carácter instrumental (pro-activo) o reactivo.

\section{VIÑETA CLÍNICA.}

A lo largo del siguiente apartado vamos a ejemplificar con un caso el trabajo realizado en un contexto de intervención familiar.

Una familia donde el espacio reflexivo queda invalidado entre los mismos sujetos, incapaces de construir un sistema promotor y potenciador de funciones mentalizadoras que favorezcan la capacidad reflexiva de los miembros de la familia. Dificultando así el desarrollo de autoconciencia reflexiva en el hijo, paciente designado por el propio sistema familiar como miembro sintomático, así como dinámicas circulares validadoras del reconocimiento de los propios estados mentales y de las del otro.

El paciente se trata de un varón de 19 años diagnosticado de Trastorno obsesivo compulsivo y en tratamiento psicofarmacológico.

El motivo de la demanda de consulta guarda relación con una relación filio-parental compleja y complicada, definida por episodios de agresividad y violencia del hijo a los padres, especialmente a la madre.

El joven pasa mucho tiempo en casa y se muestra extremadamente dependiente de su madre, denotando un carácter amable, educado, pero evidentemente tímido e introvertido, manifestando tendencia al aislamiento y susceptibilidad social. No obstante, denota mala gestión de las emociones, baja tolerancia a la frustración y mínimo control de impulsos, agresividad y descontrol en determinados momentos, llegando a la agresión a las figuras parentales durante determinados episodios de crisis.

Es necesario, para contextualizar las dificultades que denota este joven, explicar 
brevemente el marco familiar donde convive y se relaciona.

La historia vital del padre está marcada por el fallecimiento de su madre y su hermana mayor cuando él era aún un niño.

Nunca se habla de estos episodios en casa, su padre (el abuelo del joven) reprime el proceso de duelo, invalidando las muestras de tristeza y sufrimiento.

El padre invalida expresiones emocionales, en sí mismo y en el otro, aunque si se favorece un espacio de diálogo sobre ello es el primero en expresar, sobre todo tristeza, vergüenza y culpabilidad. Al inhibir dicha expresión aparece la obsesión, también en él, concretamente centrada en el orden y la limpieza, llegando a ponerse nervioso por una sábana mal doblada o un calcetín separado de su pareja.

Al igual que el hijo, muestra pensamientos obsesivos y comportamiento compulsivo, así como susceptibilidad social e irascibilidad. Unas palabras de la madre al respecto fueron: "Padre e hijo son iguales, por eso chocan tanto"

La madre del paciente describe su infancia algo desplazada en su familia por su hermana mayor. "Mi hermana siempre ha sido la preferida", "Yo siempre era la segundona". Se define a sí misma como "la oveja negra de la familia". "En esta generación me ha tocado a mí. Siempre hay alguien de mi familia que es expulsada de la misma y tiene que buscarse la vida con la familia que forma, esto me ha tocado".

La madre reconoce un miedo excesivo a la soledad, dada la perdida de relación con su familia de origen, aunque dicho miedo lo proyecta en el hijo, lo que conlleva una sobreprotección del mismo.

Respecto al hijo se muestra hiperproteccionista inhibidora infantilizando así al joven: " $A$ mi podrán decirme lo que quieran, pero a mi hijo no lo toca nadie".

En lo relativo a la historia familiar del paciente: La pareja de padres se conoce tempranamente, siendo ambos adolescentes, en este periodo ambos encuentran trabajo (él turno de noche y ella turno partido de día) además de contratos indefinidos, por lo que deciden dar un paso en la relación y se independizan, aunque no comparten intimidad dados los horarios y apenas se encuentran en la convivencia más allá de los fines de semana y vacaciones.

Durante el periodo de la primera infancia de su hijo el relato es idílico, confirmando la unión familiar, definiendo al hijo como "un niño feliz, alegre, movido. Siempre jugando, sobre todo a futbol". No obstante, la madre señala la soledad vivenciada por las noches 
con el pequeño sin el padre.

La pareja, a principios del 2000, pasa por una crisis conyugal. Esto es vivido por la madre de manera humillante y vergonzosa. "Durante ese tiempo me dijo que yo no valía para nada, que era estúpida, idiota, que estaba cansado de mí." "Me sentí humillada y maltratada".

Desde entonces, se producen una serie de acontecimientos con la familia de origen de la madre que la distancia de su propia familia. Lo que lleva a una relación de dependencia de la mujer respecto a su marido.

La relación conyugal está deteriorada, distante, no compartiendo intimidad, poca pasión y escaso compromiso.

En la relación paterno-filial, el padre se muestra distante y frio respecto al hijo, insensible a las dificultades del mismo. Invalida las expresiones de sufrimiento en la familia. Asegura que esta distancia que toma respecto a los problemas del hijo es "para garantizar la seguridad de todos, porque acabaría muy mal la cosa". Quizás se aprecia cierta celotipia paterno-filial respecto a la relación con la madre, distanciamiento entre padre e hijo debido a tal relación con la madre/esposa. Así mismo, hay una identificación proyectiva con las dificultades del hijo. "Además del nombre, ambos compartimos muchas cosas, somos iguales, con nuestras rarezas y manías, solo que yo (el padre) sé llevar sin que me afecte" "no soporto verle hacer tantas tonterías, es que parece idiota y eso me cabrea". A lo largo del proceso aparece culpabilidad: "Me siento culpable de que mi hijo sea así, creo que he sido un mal padre, lo he hecho mal y no sé qué hacer para cambiarlo".

Respecto a la relación materno-filial, observamos que se configura en torno a la infantilización del hijo, sobreprotección y dependencia del mismo a la madre (y de ella al joven). En una sesión, ante la pregunta del terapeuta: "¿lmaginas el día que estés preparada para cortar el cordón umbilical y dejarle crecer?" La madre: "No imagino el día que este pajarito vuele del nido, no sé qué haremos los dos, el uno sin el otro". Esta metáfora permite hablar del proceso de desvinculación del hijo de manera aceptable y comprensible por ambos progenitores.

Los miedos de la madre, además de una historia familiar previa, favorecen un escenario que complica el desarrollo socioemocional del joven respecto a su proyecto de vida personal, lo que Cancrini (1986) denomina desvinculación imposible, en un entorno invalidante que complica el desarrollo de funciones auto-reflexivas en el adolescente. 
Señalan el síntoma del hijo como algo que les une y les separa, mostrando la obsesión del hijo en ellos mismos, construyendo la estructura y funcionamiento invalidante familiar a través de la emergencia del síntoma en el hijo.

A lo largo del proceso terapéutico, aparece la desconfirmación del hijo desde el único reconocimiento del trastorno "es un enfermo, sin nosotros no sería nadie". Estas dinámicas impiden que el adolescente siquiera pueda pensarse así mismo, pues no obtiene de sus progenitores una confianza mínima como sujeto individuado capaz de tomar conciencia de sus propios estados mentales, y mucho menos los del otro, pues "la enfermedad está instaurada, su mente ya no le pertenece, le pertenece a la enfermedad". La intervención en estos episodios va encaminada a la señalización y el bloqueo de la desconfirmación, invitando a la madre y al padre a hablar sobre posibles sentimientos del hijo respecto de tal afirmación.

También se propone una tarea de "mirada familiar", la cual consiste en sentarse triangularmente en casa, mirándose sin poder verbalizar nada, durante unos minutos. Tras este tiempo, se propone un espacio donde cada miembro debe escribir, por una parte, que ha sentido al mirar a su familiar y por otro, que considera que ha podido sentir el otro durante esa experiencia. Es reseñable que, a través de este ejercicio, validan actitudes del hijo encaminadas al cambio.

Ante la coincidencia conyugal en el señalamiento del hijo como portador del síntoma en la familia "Este hijo nos va a destrozar", el terapeuta opta por desfocalizar el señalamiento y ampliar el malestar en la familia "Observo todo el sufrimiento que acumuláis, el desconcierto y la tensión que la situación genera a toda la familia". Invitando a que cada uno hable del sufrimiento del otro, incluido el sufrimiento del joven respecto de sus propias dificultades, promoviendo un discurso circular en el que no cabe la comunicación directa ni personal sino una narrativa de lo que otro miembro puede estar sintiendo en la dinámica familiar ciertamente disfuncional.

Durante el proceso también aparecen transacciones descalificadoras de los padres al hijo: "eres un inútil" "no vales para nada" "eres un perdedor" "eres así porque te sale de los huevos, no quieres cambiar, solo quieres joder". En estos momentos se invita a que los padres puedan verbalizar y señalar aquellos aspectos que el hijo comparte con el padre (narrado por la madre) y con la madre (verbalizado por el padre), invitando a la comunicación circular entre los miembros de la familia, mostrando así la capacidad de poder mirar más allá del sufrimiento personal e identificar aspectos positivos del hijo que guardan relación con ambos progenitores. Aquí aparece la culpabilidad parental, 
desde donde comienzan a poder hablar del sufrimiento del hijo, de lo que consideran que le está sucediendo y de la poca disposición de los padres para que el hijo pueda expresar dicho malestar.

Otra de las situaciones que suelen darse a lo largo de las sesiones familiares es la escalada simétrica paterno/filial (discusiones entre ambos que desenlazan en enfrentamientos ciertamente agresivos)

Por ello se establece una alianza terapéutica con el hijo, favoreciendo la circularidad en la comunicación: "entiendo que debes de sentirte solo y nervioso cada vez que no comprendes por qué haces lo que haces, pero además te critican tus seres queridos por ello. ¿Crees que ellos comprenden algo de lo que te sucede?, ¿Cómo crees que ellos lo sienten?, ¿Podrías explicármelo a mí? quizás así puedan escucharte, dado que hablar directamente resulta muy complicado."

Poder hablar con sus padres, de manera indirecta a través del terapeuta, le invita a verbalizar sentimientos que anteriormente quedaron invalidados dada la falta de disponibilidad de los progenitores a hablar de lo que genera sufrimiento. Esta posibilidad alivia al joven y permite a los padres escucharle de manera no directa a ellos mismos, hecho que ayuda a construir un espacio de comunicación circular donde un miembro puede hablar de los propios estados mentales y de los demás al terapeuta, pero posibilitando que los demás puedan elaborar los suyos propios diferenciados de los estados mentales del otro.

En un determinado momento, el joven quiere recordar "otro tiempo pasado". "Casi ya no recuerdo cuando era niño. Imagino que era feliz, que reía, jugaba y esas cosas. Mi padre dice que era como los demás y que incluso jugábamos juntos y reíamos. Me gustaría recordar". Ante esta proposición se invita a la familia a elaborar un Collage Familiar, recuperando fotografías, estableciendo una historia y elaborando juntos un cuadro que represente el comienzo de la familia y la niñez del hijo. Este ejercicio les permite hablar entre ellos de aquellas experiencias emocionales personales y relacionales que sugieren las imágenes recuperadas de la etapa de la primera infancia del ahora adolescente, beneficiando un espacio reflexivo validante y deslizando un discurso personal hacia una narrativa relacional y vincular, reflexiva y mentalizante.

A lo largo del proceso, el objetivo que se persigue es el desarrollo reflexivo familiar respecto de las dificultades del hijo, la pareja y la propia familia en torno a dichas dificultades, poniendo una mirada sobre su hijo más allá del síntoma, validando y reconociendo al sujeto adolescente, favoreciendo un espacio reflexivo y mentalizador 
que introduzca la posibilidad de que cada miembro pueda admitir el sufrimiento del otro, tanto desde su propio estado mental como el del familiar, favoreciendo la expresión de ese malestar en un sistema previamente invalidante. Atendiendo, transversalmente, un proceso de desvinculación sano y saludable del hijo adolescente.

\section{ALGUNAS REFLEXIONES}

Nuestra postura mantiene que existe una bidireccionalidad entre los componentes de un sistema relacional determinado. Por ejemplo, en la relación entre los padres y los hijos entendemos que hay una bidireccionalidad entre los estilos de crianza o educativos de los padres con los respectivos estilos de apego que presentan y los estilos de crianza o educativos percibidos por los hijos según sus propios estilos de apego y las experiencias de relación acumuladas en su entorno familiar. Cada uno de ellos es el estímulo relacional que genera interpretación y respuesta por parte del otro, la cual a su vez depende de las características individuales de cada miembro anteriormente mencionado. De esta forma aunque la secuencia espiral siempre se da, la forma en que se produce puede variar según la combinación de estos factores. Con ello nos referimos a la mutua influencia desmarcándonos de visiones más causales que inciden en la predominante importancia de las características del subsistema parental. En este sentido, creemos que las respuestas del subsistema parental pueden influir en la interpretación y acción del filial, pero también creemos que las respuestas del subsistema filial pueden condicionar (ratificando o invalidando según el caso) las propuestas parentales.

Así, las dinámicas relacionales contribuyen a generar un ambiente familiar que constituye un sistema que responde a un principio holístico desde el que el todo supone una entidad diferente a la suma de las partes y de sus causalidades relacionales. Así, dicho ambiente acaba influyendo a cada uno de sus componentes, que no suele ser consciente de dicha influencia. Una de las consecuencias suele ser la contribución a generar un aprendizaje, adecuado o no, en la gestión, regulación o representación mental de los estados emocionales que se encuentran tras las respuestas funcionales o conductuales que se emiten.

En el análisis de la circularidad partimos inicialmente de dos taxonomías ya existentes, una desde la perspectiva individual del sujeto joven que ejerce la conducta transgresora y/o violenta y, otra, desde el tipo de respuesta que el entorno da al comportamiento o 
conducta del joven. Micucci señala que "estas conductas abarcan un continuo y pueden ser leves, moderadas o graves según su variedad y frecuencia, así como el potencial de daño para el joven y los demás" (Micucci; 2005:212). De esta manera entendemos la problemática de estos jóvenes no como una relación causa-efecto, sino como una dinámica circular. Ello nos llevaría a establecer el foco de la intervención sobre las dinámicas relacionales y no sobre uno de los subsistemas $y$, menos aún, sobre el paciente designado y su conducta.

Acercarse a esta perspectiva de circularidad supone el intento de integrar visiones de dinámicas relacionales que generan, mantienen y desencadenan estilos relacionales determinados. Con ello, nos referimos a que es más importante centrarnos en las dinámicas y estilos relacionales más que en los actos o conductas. La expresión de un síntoma (por ejemplo, la violencia) necesita a otro, se ejerce en relación a ese otro. Incluso los actos autolesivos son expresiones de diferentes realidades intrapsíquicas que representan al otro.

La tarea fundamental en el trabajo terapéutico consiste en identificar cuáles son los patrones relacionales que se establecen, es decir, las dinámicas relacionales que generan, sostienen y/o acrecientan el síntoma. Desde este concepto de circularidad la intervención terapéutica supone la generación de un espacio interpersonal que permita la internalización de procesos intersubjetivos que modifiquen el estilo relacional que expresa un síntoma disfuncional como acto.

A modo de resumen, desde esta perspectiva que proponemos de la circularidad señalaríamos los siguientes aspectos como nucleares en la intervención terapéutica:

$1^{\circ}$ Ver la circularidad en las relaciones interpersonales dentro del espacio terapéutico. Esto es, en el análisis de las dinámicas inter-relacionales dentro de un sistema familiar y dentro del sistema terapéutico, en el que el terapeuta también está incluido. Esta perspectiva implica darme cuenta que la conducta de uno es el estímulo competente, concepto de Damasio (2005) de la conducta del otro; es decir, ver cómo nos relacionamos. Por ej.: Cuando TÚ haces " $\mathrm{X}$ " $\mathrm{YO}$ me siento " $\mathrm{Y}$ ". El primer paso consiste en aprender a ver esa circularidad, ser conscientes de la dinámica relacional que se genera. Sería pasar de "qué pasa" a "qué nos pasa". Este aspecto implicaría el paso de la definición del problema centrado en el síntoma (en el paciente identificado) a una definición relacional del problema.

$2^{\circ}$ Cuando se han observado las circularidades en los dos niveles dentro del sistema con el que nos relacionamos, hay que elegir dónde se puntúa. El punto de arranque no tiene 
que ser siempre el síntoma del paciente identificado. Nosotros proponemos elegir a la persona "más capaz" para puntuar la historia o secuencia desde él. La persona "más capaz" para poder entenderlo, aceptarlo sería también la persona más sensible, con una mayor capacidad de insight y de mentalización.

$3^{\circ}$ Desarrollar un "yo observador". Lo que se va a observar no es lo que hace el paciente identificado solamente (el síntoma), el gran esfuerzo que tenemos que hacer es colocarnos fuera de la díada, rescatar un yo observador que mire qué es lo que está pasando entre los dos, qué tipo de vínculo se constituye". Detectar los roles recíprocos, detectar dónde nos pone el paciente o la familia en esos patrones relacionales y no dejar que nos coloquen ahí para evitar entrar en un isomorfismo con la familia,

Para nosotros los conceptos de circularidad y mentalización se encuentran interrelacionados. En este sentido, el desarrollo de la comprensión del estado mental está integrado dentro del mundo social de la familia, con su red compleja de relaciones. Así mismo la naturaleza de las experiencias infantiles influye en el desarrollo de la mentalización. La circularidad bidireccional de las relaciones emocionales del niño con los padres ocupa un rol central en el desarrollo de la habilidad para comprender la interacción social en términos psicológicos. De esta manera podríamos considerar que la mentalización implica focalizar en los estados mentales de uno mismo y de los otros, en especial al explicar la conducta con base en los pensamientos, sentimientos, creencias y deseos que determinan lo que hacemos. En otras palabras, se trataría de interrelacionar el mundo intrapsíquico de cada miembro de un sistema familiar y el mundo interpersonal, es decir, las dinámicas relacionales entre los progenitores y el hijo adolescente.

No se trata de un abordaje individual de estos déficits (bien en las capacidades mentalizadoras, bien en la autorregulación emocional, etc.) sino que proponemos generar, habilitar dinámicas relacionales que permitan que el propio sistema valide emociones y, a su vez, que como clima permita que cada elemento del sistema optimice sus capacidades de mentalización individual. Teniendo en cuenta que la terapia parte de la base de poder poner palabras a aquello que haciéndonos sufrir no es digerible, elaborable, accesible a la conciencia. Así partimos de Bowlby para quién la primera tarea ha de ser: "Proveer al paciente de una base segura, desde la cual pueda explorar los múltiples aspectos desdichados y dolorosos de su vida, pasados y presentes, en muchos de los cuales encuentra difícil o quizás imposible pensar y reconsiderarlos sin un compañero confiable que le provea apoyo, aliento, simpatía y, en ocasiones, orientación" (Bowlby, 1988, pág.138). Un sistema terapéutico tiene que ser capaz de centrarse en el desarrollo 
de estas capacidades que posibiliten posteriormente el abordaje de ese síntoma diana, en ocasiones con el mero desarrollo de las capacidades mentalizadoras se minimiza el síntoma diana.

De esta forma, la capacidad de mentalizar, de verme y ver a los otros posiblemente contribuye al aseguramiento del estilo de apego, al desarrollo de respuestas sensibles, a la autorregulación emocional y a la tolerancia al malestar. Se construyen narrativas que permitan la confrontación o connotación positiva, cuando sea el caso, "darse cuenta" de los propios estados mentales y de los del resto de los miembros del sistema que se encuentran tras las conductas que originan malestar, "comprender" la interconexión entre los estados emocionales de los diferentes miembros y cómo la mala interpretación de los mismos puede ocasionar problemas de comunicación en la base de la disfunción familiar.

Así las cosas, aplicar el concepto de mentalización dentro de una comprensión sistémica conlleva una diferencia a cuando se hace en encuadres individuales de otras corrientes. De hecho, la dinámica desde los terapeutas consiste en generar un clima o ambiente dentro del sistema que genere la posibilidad entre los miembros de "verse" más allá de los síntomas o conductas, completar la interpretación de las mismas y abrir nuevas vías de comunicación. Tal y como el mismo Asen (2011) señala el niño que es mejor comprendido, comprenderá mejor a sus padres, las interacciones resultantes serán fácilmente comprendidas por los padres, quienes, a su vez, mejorarán las capacidades de mentalización del niño. Se puede observar una visión circular, no basada en el problema sino en las dinámicas generativas y positivas de desarrollo como prevención o componente paliativo de posibles problemas. Posibilita, a su vez el desarrollo de insight y de poner palabras, necesario en la intervención terapéutica. En la devolución que hagamos, después de la entrevista familiar de valoración habría que reflejar a la familia nuestra intuición sobre su funcionamiento interpersonal y su capacidad de mentalización para que empiecen a reflexionar sobre su mente y sus dinámicas relacionales, es decir, su manera de pensar, funcionar y relacionarse.

Desde este enfoque proponemos simultanear lo individual y dimensional con lo relacional y ello conlleva establecer el foco de la intervención sobre las dinámicas relacionales que se dan entre los miembros familiares y desde los estados emocionales de los pacientes. Así las cosas, resulta fundamental generar y proponer narrativas circulares que validen simultáneamente los contenidos emocionales de los miembros del sistema, así como que confronten los contenidos compartidos con el grupo (es decir equilibrarlos, no siendo exageradamente presentes o ausentes) y cuestionen las formas 
relacionales que mantienen como sistema y que contribuyen a desregularles y/o invalidarles individualmente. Hacer narrativas que incluyan todas las emociones de cada uno de los miembros, estableciendo circularidad, es decir, relacionando que cada una de ellas se da como reacción ante la conducta del otro, que a su vez tiene una emoción por detrás.

La labor del profesional se centrará en ayudar a la familia a dar sentido a los sentimientos que son experimentados por cada miembro individual, a reconocer los pensamientos que están conectados con estos sentimientos, cómo estos sentimientos se comunican dentro de la familia (cuál es el patrón relacional familiar) y cómo la falta de comunicación o la falta de comprensión de estos sentimientos conduce a interacciones que contribuyen a incrementar o mantener los problemas familiares. El profesional realiza un trabajo exploratorio para favorecer la activación de la mentalización en la familia y cada miembro individual y supone la construcción de un equipo de trabajo familia-terapeuta. Así el profesional estimulará la participación activa y el protagonismo de la familia, así como sus puntos de vista sobre distintos aspectos del proceso terapéutico (incluidas las intervenciones del profesional). El terapeuta utilizará aquellas intervenciones que favorezcan que los miembros de la familia dirijan su atención hacia sí mismos, los contenidos de su mente, su modo de funcionamiento mental, los estados mentales de los otros significativos y la interrelación que se genera entre ellos

Las estrategias terapéuticas se centran en el desarrollo y fortalecimiento de la capacidad de mentalización en la familia, pretenden crear una matriz mentalizadora en la familia que les permita comprender y dar sentido a las conductas relacionadas con el síntoma motivo de consulta.En el transcurso del proceso terapéutico resulta necesario estar monitorizando los estados mentales de los pacientes a la vez que autoobservamos nuestro propio estado mental (desarrollo del "yo observador" por parte del profesional). En caso de duda o peligro de intensidad emocional excesiva bajaremos a niveles de intervención más bajos, utilizando aquellas técnicas terapéuticas que impliquen una menor activación emocional. Tenemos que regresar al momento en que las personas se sentían seguras y confiadas y así favorecer el tránsito de un discurso no mentalizador a un discurso mentalizador. Cada intervención debe ajustarse al nivel de mentalización que presenta la persona en ese preciso momento.

\section{ALGUNAS TÉCNICAS}

Con el fin de fomentar la mentalización de cada miembro de la familia, toda una gama 
de diferentes técnicas de mentalización se puede emplear (Asen y Fonagy, 2011; Fearon et al, 2006). A continuación, vamos a exponer una serie de técnicas de mentalización coherentes con la práctica sistémica y ordenadas de menor a mayor activación emocional:

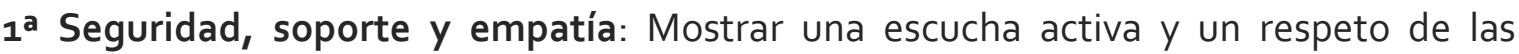
vivencias de los miembros de la familia. Esta técnica se asemeja a los procesos de unión y acomodación ("joining") con la familia que plantea la escuela estructural, tal y como explica el propio S. Minuchin:

"...requiere la acomodación del terapeuta a la familia para formar un sistema terapéutico seguido por su evaluación de sus experiencias de la interacción de la familia en el presente...".

"La familia se modifica solamente si el terapeuta ha logrado incorporarse al sistema de un modo sintónico a éste" (Minuchin, 1977. p.185).

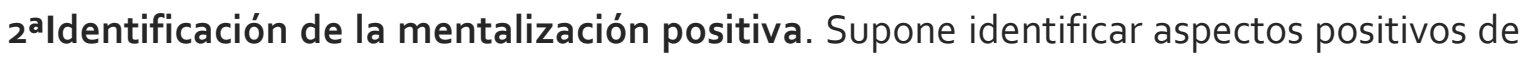
su capacidad de mentalización (de cada miembro del sistema familiar) y relacionarlos con el propósito de la terapia.

Mediante esta técnica generaremos curiosidad en los miembros de la familia acerca de sus motivaciones, demostrando que la comprensión de los estados mentales propios y ajenos nos ayuda a controlar mejor nuestra emoción.

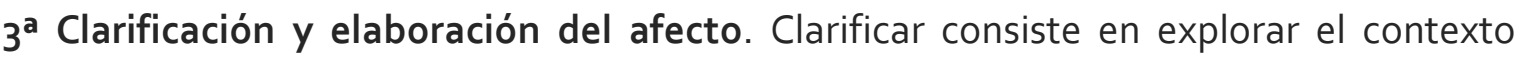
interpersonal en que se dio una conducta problemática (o síntoma), resultado de un fallo en la capacidad de mentalización. Intentaremos, por un lado, conectar los actos con las emociones que ha sentido cada persona $y$, por otro lado, interrelacionaremos los comportamientos de cada miembro presente en esa dinámica relacional generando una narrativa circular desde sus estados emocionales. Ello parte, a su vez, de la capacidad del profesional de especular individualmente el estado mental subyacente de cada miembro del sistema y encontrar una narrativa que encuentre la conexión relacional entre los mismos desde dichos contenidos emocionales y expresados en conductas. La exploración del afecto ayuda a prevenir la sobrecarga emocional y, por tanto, la pérdida de mentalización.

$4^{\text {a }}$ Detenerse y confrontar. Consiste en parar y ayudar al paciente a que focalice en cómo ha sido el cambio de su estado mental. 


\section{$5^{\mathrm{a}}$ Mentalización básica:}

5.1.) Parar, escuchar y mirar. Consiste en realizar una exploración activa sobre lo que sienten y piensan los pacientes durante la sesión. Se trata de parar una dinámica relacional disfuncional y llevarles a reflexionar sobre lo emocional, que se den cuenta no sólo de qué están sintiendo tanto cada uno de ellos como el otro sino de la interrelación existente entre sus estados emocionales; es decir, la dinámica circular que se genera desde esos estados emocionales, dinámica de la que ellos no son conscientes y que contribuye a generar ese ambiente disfuncional por el que todos están de uno u otro modo afectados.

Esta técnica respeta el ritmo de los pacientes, es decir, se pretende ir acostumbrando a los pacientes a reflexionar sobre estas emociones, pero muy poco a poco, sin romper el apego y respetando su ritmo.

\section{2.) Parar, rebobinar y explorar.}

El discurso tiene que ser desplazado de no-mentalización a mentalización, rebobinando sobre los propios pasos a donde la mentalización fue evidente por última vez. El relato de la familia puede entonces comenzar de nuevo desde ese punto.

Esta técnica de mentalización resulta similar a la técnica de "la moviola" que se utiliza en el enfoque post-racionalista (V. Guidano).

6a Mentalizar la relación terapéutica. Consistiría en mentalizar las dinámicas relacionales, el vínculo que se da entre los distintos miembros del sistema incluyendo al profesional. Así mismo desde este enfoque la relación terapéutica no se establece en la transferencia, sino que lo que ocurre en el sistema terapéutico tiene que ser entendido en la relación conmigo, como profesional y parte de ese sistema. Pasaríamos a hablar de lo que Abeijón (2013) denomina el "espacio relacional terapéutico", alentando a los miembros familiares a:

- Reflexionar sobre su relación actual.

- Enfocar su atención en otra mente.

- Contrastar su percepción de sí mismo con la percepción que otras personas significativas tienen sobre ellos.

Atendiendo a la evaluación del funcionamiento interpersonal y la capacidad de mentalización que hayamos realizado previamente tanto del sistema familiar como de cada miembro que lo compone valoraremos la conveniencia de iniciar esta técnica en la 
relación propiamente terapéutica, esto es, entre un miembro determinado y el profesional; para con posterioridad, posibilitar que el miembro más capaz del sistema familiar la realice con otro miembro familiar.

Otras técnicas que contribuirían a facilitar la mentalización en el espacio relacional terapéutico serían:

- La metacomunicación: sería la explicación que nos damos y que solicitamos al otro, acerca de nuestras conductas -y las de nuestro interlocutor-y sus efectos en la relación mientras estamos interactuando. Se trata, en definitiva, de una comunicación acerca de una comunicación. Nos permite colocarnos fuera del marco de la interacción -como contexto-, para reflexionar y vernos en la relación.

- Preguntas vinculadas a lo somático que ayuden al paciente a ir poniendo nombre a sus sensaciones físicas para darle una representación mental de esa emoción.

- Las técnicas del equipo reflexivo aumentan la fuerza de la mentalización.

- La técnica de la entrevista circular y reflexiva tiene como efecto generar múltiples perspectivas. Las técnicas de intensificación del stress mediante la alteración en el espacio físico que propone Minuchin. Tal y como explica Asen (2011) estas técnicas hacen que familias de no mentalización sean accesibles a la intervención terapéutica a través de una alteración en el dominio físico, porque esta alteración tiene consecuencias palpables en las experiencias subjetivas de la familia. Reflexionando sobre estas consecuencias, inevitablemente, provocará (fortalecer) la mentalización. Este es el beneficio oculto de la creación de nuevas perspectivas y formas de ver el otro.

- Asen propone el bucle de la mentalización como un mapa de ruta. Se trata de una herramienta para el cambio con cinco posiciones diferentes de mentalización que el terapeuta toma: puntuando, controlando, mentalizando el momento, generalizando y revisando. Este autor habla de bucle, ya que no es una progresión lineal de etapas sucesivas, sino un proceso recursivo de observar, comprobar, revisar y hacer nuevas observaciones. En la primera posición, el terapeuta hace una declaración provisional (puntuando) acerca de una interacción entre los miembros de la familia que ha observado en el aquí y el ahora de la sesión. El terapeuta identifica y pone de relieve una interacción que parece estar relacionada con alguna dificultad para la mentalización. A continuación, invita a la familia y sus miembros individuales para conectar con 
ello, pero también dando la posibilidad de que lo desestimen. Si existe cierto reconocimiento entre los miembros de la familia y se comprometen con la observación del terapeuta se toma la segunda posición de mentalizar el momento. El terapeuta modela una postura de mentalización, que muestra el respeto y la curiosidad por las mentes de otros. Esta actitud transmite la idea de que aprender sobre cómo otras personas están pensando y sintiendo. Esta invitación para llevar a cabo algún tipo de brainstorming (lluvia de ideas) emocional anima a los miembros de la familia a poner voz a los sentimientos. El terapeuta intenta ayudar para generalizar alejándose de la discusión específica y ampliar su punto de vista. Los miembros de la familia están invitados a elaborar algunas observaciones de carácter más general y reflexionar sobre cómo los patrones de interacciones similares tienden a evolucionar de forma espontánea en el hogar y los estados emocionales que estos provocan. En un intento por identificar y abordar las situaciones de problemas típicos, lo que se ha observado en el aquí y el ahora de la sesión se enlaza con las situaciones de la vida real. El terapeuta anima activamente a los miembros de la familia a etiquetar sus propios sentimientos, para reflexionar sobre lo que debe ser para ellos. En una etapa posterior, a menudo hacia el final de la sesión, el profesional querrá revisar lo que esta experiencia ha sido para todo el mundo. Aquí se mira hacia atrás y comprueba los estados emocionales de cada miembro de la familia. Esto ayuda tanto al profesional como a la familia para evaluar cómo una experiencia nueva y cargada de emoción se ha registrado en los diferentes individuos y proporcionado una oportunidad para reflexionar juntos sobre lo sucedido y sus posibles consecuencias. En esta última posición se promueve una reflexión conjunta entre todos de lo que pasa en la cabeza de todos. La terapia familiar basada en la mentalización (MBT-F) incluye una serie de actividades de mentalización, que son presentadas como juegos o tareas que la familia puede llevar a cabo (Manuales Tiddly, 2010). Estas tareas principalmente funcionan como rompehielos, creando una aclimatación gradual a la mentalización en las familias en las cuales experiencias pasadas han creado una preocupación implícita o incluso una evitación fóbica de la actividad. Las tareas también tienen una función de desarrollo de habilidades, así como el potencial de generar información relevante (perspectivas alternativas). La tarea también puede ayudar con la generalización del aprendizaje como parte de los deberes.Asen propone diversas actividades de mentalización tales como la inversión de los papeles, el juego de la sensación del buscador en que se invita a los miembros de 
la familia a crear una historia centrada en los sentimientos que experimentan, la actividad del botón-pausa de pensamiento y el juego de la exploración cerebral que suele finalizar con una discusión sobre cómo cada uno puede parecer leer con precisión los estados mentales y el hecho de que nunca se puede saber completamente lo que otros piensan y sienten.

\section{CONCLUSIONES}

Hemos tratado de exponer un enfoque integrador basado en un concepto de circularidad que incluye la interrelación entre el mundo intrapsíquico y el mundo interpersonal. Desde este enfoque cobra especial relevancia la inclusión de la mentalización, concepto que incluye tanto la visión de mí mismo como la visión de los otros sobre mí (modelos operativos internos) y que ayuda a comprender y fortalecer la interrelación existente entre el mundo interno y externo, promoviendo la responsabilización entre los distintos miembros que conforman el sistema terapéutico (incluído el profesional).Asimismo la inclusión de las técnicas de mentalización contribuye a mejorar la eficacia de la terapia familiar, haciendo a los miembros de la familia más receptivos a la sintonización con el pensamiento y los sentimientos de los demás.

Es por ello que proponemos la necesidad de un cambio en el rol profesional y la importancia del trabajo personal del profesional (a través de espacios de supervisión, trabajo en equipo, terapia, etc.) que contribuya al desarrollo del "yo observador" por parte del profesional. Por otra parte, cada vez más se subraya como factor positivo para el éxito de la terapia favorecer una mayor participación de los pacientes en su proceso terapéutico, ya que éstos son los verdaderos protagonistas del cambio.

Tanto la mentalización, como el enfoque intersubjetivo y las corrientes sistémicas suponen nuestras bases de concepción y construcción de una propuesta metodológica de intervención en contextos de involuntariedad con una perspectiva psicoterapéutica de apoyo. Los elementos conceptuales y técnicos que se repiten en las citadas psicoterapias constituyen la base de factores comunes que sustentan nuestra mirada integradora. 


\section{REFERENCIAS}

Abeijón, J.A. (2007). María está enfadada. Terapia familiar desde Iberoamérica. Buenos Aires. Edit. Treshaches.

Abeijón, J.A. (2013). Patología en la demanda. Mosaico. 54: 62-75.

Allen, J.G. y Fonagy, P. (2006).Handbook of Mentalization-Based Treatment.JohnWiley\&Sons, Ltd.

Asen, E. y Fonagy, P. (2011).Mentalization-based Therapeutic Interventions for families. Journal of FamilyTherapy, 2011.

Bateman, A, Fonagy, P. (2008). Comorbid antisocial and borderline personality disorders: mentalization based treatment. Journal of Clinical Psychology: in session, 64: 1-14.

Bateman, A.; Bolton, R.; Fonagy, P. (2013). Antisocial personality disorder: a mentalizing framework. Focus: Journal of Lifelong learning in Psiquiatry. XI: 1-9.

Bateman, A.; Fonagy; P.; (2016). Mentalization based treatment for personality disorder: Apractical guide. Chapter 13. Antisocial personality disorder. OUP: Oxford.

Bleiberg, E. (2013).Mentalizing-based treatment with adolescents and families. Child and adolescents Psychiatric Clinics of North America, 2 (2)295-330.

Bleichmar, E. D. (2005).Manual de psicoterapia de la relación padres e hijos. Barcelona, Ed. Paidós.

Bowlby, J. (1998).El apego. Barcelona. Ed. Paidós.

Bowlby. J. (2006). Vínculos afectivos: Formación, desarrollo y pérdidaEd.Morata

Bowlby. J. (1989). "Una base segura, aplicaciones cínicas de la teoría del apego" Ed. Paidós, Psicología Profunda

Caillé. P. (1992). Uno más uno son tres, la pareja revelada a si misma. Ed Paidós, Terapia Familiar.

Cloninger. S. Teoría de la personalidad Ed. Pearson

Damasio, A. (2005). En busca de Spinoza. Barcelona.

Estalayo, A. (2011). Violencia adolescente y circularidad: de la teoría a la práctica, Cap. XX en Adolescentes en el siglo XXI. Entre impotencia, resiliencia y poder, Roberto Pereira (Comp.), Madrid, Ed. Morata. Págs. 385-402.

Estalayo, A.; Rodríguez O. Y Romero J.C. (2009). Estilos de crianza y ambientes familiares en menores y jóvenes violentos. Un modelo psicoterapéutico de intervención. Cuadernos de Psiquiatría y Psicoterapia del niño y del adolescente nº 33/34.

Fonagy, P. (1999). Persistencias transgeneracionales del apego: una nueva teoría.Aperturas psicoanalíticas. Revista Internacional de Psicoanálisis nº 3.

Fonagy, P. (1999). Apegos patológicos y acción terapéutica.Aperturas psicoanalíticas. Revista 
Internacional de Psicoanálisisn ${ }^{\circ} 4$.

Fonagy, P.; Target, M. (1999). Towards understanding violence: The use of the body and the role of the father. In R. Perelberg (ed.) Psychoanalitic understanding of violence and suicide. London: Routledge. (pp.53-71).

Fonagy. P.Teoría del apego y psicoanálisis. Barcelona: ESPAXS

Fonagy, P. \& Allison, E. (2014).The role of mentalizing and epistemic trust in the therapeutic relationship .Psychotherapy 51, 372-380.

Lanza Castelli, G.Mentalización y expresión de los afectos: un aporte a la propuesta de Peter Fonagy, Revista aperturas psicoanalíticas nº 031

Lanza Castelli, G. La mentalización, su arquitectura, funciones y aplicaciones prácticas.

Lecannelier, F. Psicoterapia infanto-juvenil: una aproximación integracionista desde el apego, la mentalización y la regulación emocional. Centro de Estudios Evolutivos e Intervención en el Niño, Universidad del Desarrollo.

Linehan, M. M. (2003). Manual de tratamiento de los trastornos de personalidad límite, Barcelona: Paidós.

Marrone. M. (2001). La teoría del apego un enfoque actual. Ed. Psimática

Micucci, J.A. (2005). El adolescente en la terapia familiar. Cómo romper el ciclo del conflicto y el control. Buenos Aires:Amorrortu.

Minuchin, S. y Fishman, H. Charles (1984). Técnicas de terapia familiar, Buenos Aires: Paidós Terapia Familiar.

Rioseco, S., Vicente P., Saldivia, B.,Cova, S.,Melipillán, A., Rubi, G. (2009). Prevalencia de trastornos psiquiátricos en adolescentes infractores de ley. Estudio caso-control, Revista Chilena de Neuropsiquiatría, vol. 47, núm. 3

San Miguel, T. (2009). Regulación emocional, mentalización y constitución del sí mismo.Aperturas psicoanalíticas. Revista Internacional de Psicoanálisis $\mathrm{n}^{\circ} 29$.

Sluzki, C. (2009). Bebés difíciles, progenitores difíciles hacia un modelo basado en la calibración recíproca. REDES nº 22, págs.11-27.

Stolorow, R.D. y Atwood, G.E. (2004). Los contextos del ser. Las bases intersubjetivas de la vida psíquica. Barcelona: Ed. Herder.

Stolorow, R, Orange,D. y Atwood G. (2012). Trabajando intersubjetivamente. Contextualismo en la práctica clínica. Madrid: Ágora relacional.

Viloria, I.; Ballespí, S. (2016). El papel de la mentalización en la etiología de la conducta violenta. Psicopatología y Salud mental del niño y del adolescente 2016, 27, 91-100.

Vygotski, L. S. (1978). El desarrollo de los procesos mentales superiores, Barcelona: Biblioteca de Bolsillo.

Watzlawick. P. (1997). Teoría de la comunicación humana, interacciones, patologías y paradojas. Barcelona: Ed. Herder. 
Yakeley, J. (2009). Working with violence. A contemporary psychoanalytic approach. ISBN 9780230203631

Original recibido con fecha: 6-11-2016 Revisado: 15-1-2017 Aceptado: 28-2-2017

(c) Derechos reservados/Copyright de Clínica e investigación Relacional y los autores. Prohibida la reproducción total o parcial sin autorización expresa. Este material es para uso científico y profesional exclusivamente y puede contener información clínica sensible. Los editores no se responsabilizan de los contenidos de los autores. Dirigir las consultas sobre derechos y autorizaciones a ceir@psicoterapiarelacional.es 